\title{
Inter-trial Reliability, Cardio and Demographic Markers of Rey Auditory Verbal Learning Test Performance in Young Adult Sample from Nigeria
}

\author{
Valentine Afamefuna Ucheagwu $^{1}$, Rita Nonye Ugokwe-Ossai ${ }^{2}$, Uche Okpaleke ${ }^{1}$, Jesse Ugokwe ${ }^{3}$ \\ ${ }^{1}$ Department of Psychology, Chukwuemeka Odumegwu Ojukwu University, Igbariam, Nigeria \\ ${ }^{2}$ Department of Psychology, Nnamdi Azikiwe University, Awka, Nigeria \\ ${ }^{3}$ Department of Psychology, Imo State University, Owerri, Nigeria
}

Email address:

afamval@yahoo.com (R. N. Ugokwe-Ossai)

\section{To cite this article:}

Valentine Afamefuna Ucheagwu, Rita Nonye Ugokwe-Ossai, Uche Okpaleke, Jesse Ugokwe. Inter-trial Reliability, Cardio and Demographic Markers of Rey Auditory Verbal Learning Test Performance in Young Adult Sample from Nigeria. American Journal of Psychiatry and Neuroscience. Vol. 5, No. 6, 2017, pp. 70-77. doi: 10.11648/j.ajpn.20170506.13

Received: September 28, 2017; Accepted: October 23, 2017; Published: November 29, 2017

\begin{abstract}
Objectives: The current study examines the inter-trial reliability as well as cardio and demographic markers of Rey Auditory Verbal Learning Test (RAVLT) performances in Nigeria young adult sample. Methods: Young adults between the ages of $22-40$, were enrolled for the study. They included healthy controls $(\mathrm{HC}, \mathrm{n}=102)$ and psychiatric group $(\mathrm{PG}, \mathrm{n}=25)$ who completed the serial test protocols of RAVLT as well few indices of cardio status: Blood pressure (systolic/diastolic) and pulse rate. Results: Correlation analysis showed significant positive inter-trial correlations among trials $1-5$ of RAVLT. When the immediate post interference and delayed trials were built into the analysis, positive significant relationships were also found. However no significant relationships were found on time taken to complete each trials of RAVLT except on few trials. Regression analyses showed low predictive strength of systolic and diastolic pressures and pulse rate on total performance score of RAVLT (Trials $1-5$ ) as well as the delayed trial score. However the pulse rate showed strong predictor of Recall Word List (RWL) trial of RAVLT. Analysis of covariance showed significant group differences in all trials of RAVLT except the distraction trial. Equally gender differences were found on total trial, distraction trial and delayed trial respectively. Conclusion: The study was the first to examine some psychometric properties of RAVLT in Nigeria sample and highlights good inter-trial reliability and discriminant validity of the test. It further highlights the possible roles of cardio variables on episodic memory in young adults and may have implications for treatment among this group.
\end{abstract}

Keywords: RAVLT, Reliability, Episodic Memory, Psychiatry, Validity

\section{Introduction}

The Rey Auditory Verbal Learning Test (RAVLT) is an efficient neuropsychological instrument for evaluating episodic declarative memory [1]. It provides scores for assessing immediate memory, new verbal learning, susceptibility to interference (proactive and retroactive), and retention of information after a period of time and memory recognition [2], [3], [4]. The RAVLT is sensitive to neurological impairment and verbal memory deficits in a variety of patient groups [5], [3]. Some studies have shown various patient groups sensitive to RAVLT including left temporal epilepsy [6], [3], schizophrenia [7], Parkinson disease [3], autism [8], depression [3] and dementias [9], [3].

The sensitivity of the RAVLT contributes to its clinical utility and ability to make clinical distinctions between neurological disorders. Studies have further demonstrated strong correlations of verbal learning and verbal episodic declarative memory of the RAVLT and measures of executive functions [19], [11], [9]. The present study investigates some psychometric properties of RAVLT among young adult samples from Nigeria. It further examines the cardiovascular, demographic and mental health status as determinants of RAVLT performances in the sample population. 
RAVLT performances can be affected by demographic variables including age, gender, education, intelligence level and cultural influences [2], [5], [4], [1]. Magalhaes and Hamdan [1] showed significant correlation between RAVLT performance and age and education, but not between RAVLT performance and gender. Using analysis of variance estimations, they showed significant effects of age on all RAVLT measurement and significant effects of years of education on all measurements with the exception of recognition. Among the demographic variables studied, no consensus was reached about the magnitude of the influence of these factors, although the influence of age has been suggested to be more pronounced than those of education and gender [5], [3]. Vander Elst et al [4] found that age was the strongest predictor of performance on all RAVLT scores and also showed that females and highly educated participants scored significantly higher than males and their less educated counterparts on most measurements. Mitrushina et al [5] performed a meta analysis of the roles of demographic variables on RAVLT performance. Their findings showed that age had consistent effect on RAVLT performance but unclear was the effect of education level, suggesting that this factor did not significantly contribute to the results. Intelligence level was also associated with RAVLT performance.

Conversely, de la Torre [12] hypothesized that Alzheimer disease (AD) can be viewed as a predominantly vascular disorder. The hypothesis was based on several converging lines of evidence including (i) epidemiological studies (showing that AD shares with cardiovascular disease a number of risk factors (from hypertension to diabetes) and virtually all reported risk factors for $\mathrm{AD}$ lower cerebral perfusions (ii) detection of regional cerebral hypo perfusion with the use of neuroimaging techniques is capable to identify $\mathrm{AD}$ candidates preclinically (iii) the presence of regional brain micro vascular abnormalities appear before hypometabolism, cognitive decline and neurodegenerative changes (iv) clinical overlap between $\mathrm{AD}$ and vascular dementia (v) improvement of cerebral perfusion is obtained from drugs (such as cholinesterase inhibitors) used to reduce the symptoms of AD in patients who responded to treatment [13], [14], [15]. Evidence supporting the vascular hypothesis of $\mathrm{AD}$ has been reinforced and extended in the last decade [16]. According to Pizza, Agresta, D'Acunto, Festa and Capasso [17], brain and vessel premature aging share similar molecular, cellular and tissue mechanisms such as abnormal intracellular calcium handling, epigenetic DNA damage and low level systemic and local inflammation with altered redox state.

Picano et al[ 16] made a simplified description of links between cardiovascular disease and cognitive impairment. According to them normal brain perfusion requires that all rings of the physiological chain be intact, since a chain can only lift the weight that its weakest link can bear. Thus they identified seven distinct segments in series contributing to normal perfusion with well oxygenated blood in the healthy aging brain. The segments included: the lungs, the hearts, the aorta, the baroflex, the cerebrovascular arteries, the small cerebral vessels, and the cerebrospinal venous system. An anatomic or functional alteration of each segment can lead to impaired cerebrovascular perfusion and increased risk of dementia, although frequently these conditions are not isolated and are often mutually interrelated [16]. On the other hand, blood pressure is a measurement of the force applied to the walls of the arteries as the heart pumps blood through the body. The pressure is determined by the force and amount of blood pumped as well as the size of flexibility of the arteries. A high blood pressure (hypertension) can put extra strain on arteries and may lead to cardiovascular diseases. Very many research findings showed that increase in blood pressure are associated with incremental reductions in cognitive functions [18], [19], [20].

In Nigeria, researches on neuropsychological assessment have been sparingly conducted and no normative data is available for clinical assessment. Subsequently demographic assessment of RAVLT as a screening tool has not been researched in Nigeria as well as looking into RAVLT as a discriminative tool for psychotic depression and normal controls. In addition to this, global researches into blood pressure as it relates to cognitive impairment have been done among the mid to late adults with little studies in the early adult group. Based on these problem statements, the following research questions were raised: (i) will blood pressure be strong predictor of RAVLT performance among young adults (ii) will gender, mental health status and age (as covariate) differ significantly among young adult sample in RAVLT performance respectively(iii) what are some possible psychometric properties of RAVLT in Nigeria young adult sample.

\section{Method}

\subsection{Participants}

One hundred and twenty seven participants between the ages of $22-40$ years were enrolled for the study. The participants were 61 males and 66 females enrolled from two centres. They were divided into 2 groups: Healthy Control (HC) and Psychiatric Group (PG). The HC were enrolled through advert at Psychology Department Chukwuemeka Odumegwu Ojukwu University while the PG was recruited from inpatient Psychiatric Rehabilitation Centre in the same state with the University. The psychiatric participants were diagnosed of psychotic disorder with depressive features. Their diagnoses were made by the clinicians in the centre. Both groups had atleast 14 years of education.

Table 1 shows the demographic data of the participants. Significant age differences existed among two independent variables (gender and group) in the sample population. The psychiatric group and female gender showed higher age scores compared with the normal group and male gender samples respectively. 
Table 1. Descriptive statistics of the sample population.

\begin{tabular}{|c|c|c|c|c|c|c|}
\hline Variables & $\mathrm{N}(\%)$ & $\overline{\bar{X}}$ age & Std Error & Std & $F / \mathbf{X}^{2}$ & Sig. \\
\hline Gender: Male & $61(48.03 \%)$ & 27.91 & 0.50 & 5.65 & 17.56 & 0.001 \\
\hline Female & $66(51.96 \%)$ & 31.23 & 0.61 & 6.47 & & \\
\hline Group: HC & $102(80.31 \%)$ & 23.04 & 0.34 & 1.99 & 274.04 & 0.001 \\
\hline PG & $25(19.69 \%)$ & 36.09 & 0.71 & 7.38 & & \\
\hline Gender * & Group & & & & & \\
\hline Male(HC) & $45(73.77 \%)$ & 23.20 & 0.51 & 2.00 & & \\
\hline Male (PG) & $16(26.23 \%)$ & 32.63 & 0.85 & 6.80 & 21.16 & 0.001 \\
\hline Male Total & 61 & 25.67 & 0.45 & 5.66 & & \\
\hline Female(HC) & $57(86.36 \%)$ & 22.88 & 1.14 & 2.00 & & \\
\hline Female (PG) & $9(13.64 \%)$ & 39.56 & & 6.50 & & \\
\hline Female Total & 66 & 25.16 & & 6.47 & & \\
\hline
\end{tabular}

\subsection{Instrument}

Rey Auditory Verbal Learning Test (RAVLT) and digital sphygmomanometer were used as instruments for the study. RAVLT affords an analysis of learning and retention. It involves a five - trial presentation of a 15 - word list (list A), a single presentation of an interference list (list B), two post interference recall trials (one immediate, one delayed) and recognition of the target words presented with distracters (RWL) [21]. Rey Auditory Verbal Learning Test has been shown to be reliable and valid in neuropsychological assessment of memory in various disorders including multiple sclerosis [22], Huntington's disease [23], Alzheimer type dementia [24], [25], mild cognitive impairments at risk for dementia [26], [27]. The digital sphygmomanometer was used to measure the blood pressure and pulse rate per minute of the participants.

\subsection{Procedure}

The participants (Healthy Control) were recruited based on adverts placed around the faculty of social sciences (department of psychology) of the university. The psychiatric group (PG) was recruited based upon the availability and diagnosis of the patients as well as informed consent from their primary care givers and them as well. The HC was tested at the psychology laboratory under conducive learning environment while the PG was tested at the rehabilitation centre. The tests were conducted by an expert neuropsychologist blinded on the research procedures and designs to avoid bias. Resting blood pressure and pulse were first measured after 5 minutes of rest in supine position before the administration of the RAVLT on the same day. The standard protocol for administration of RAVLT was followed [21].

\subsection{Design and Statistics}

The design of the study was a quasi experimental factorial design. It was quasi because extraneous variables like intelligent quotient, developmental and educational experiences were not controlled. The statistics was a 2 WAY ANCOVA statistics, multiple regression analysis and correlation statistics.

\section{Results}

\subsection{Inter Trial Correlations}

Inter trial correlations were performed to determine the level of inter trial correlations among the RAVLT trials. The initial correlations were among the first five trials. The findings showed significant correlations of the trials ranging from 0.52 to 0.74 .

Table 2. Correlation Table of RAVLT Trials 1 - 5 .

\begin{tabular}{llllll}
\hline Source & Trial 1 & Trial 2 & Trial 3 & Trial 4 & Trial 5 \\
\hline Trial 1 & 1.00 & $0.67^{* *}$ & $0.66^{* *}$ & $0.57^{* *}$ & $0.58^{* *}$ \\
Trial 2 & & 1.00 & $0.65^{* *}$ & $0.60^{* *}$ & $0.52^{* *}$ \\
Trial 3 & & & 1.00 & $0.75^{* *}$ & $0.71^{* *}$ \\
Trial 4 & & & & 1.00 & $0.71^{* *}$ \\
Trial 5 & & & & & 1.00 \\
\hline
\end{tabular}

$* *=$ Correlation is significant at 0.01 level.

Additionally when immediate post interference trial and delayed trial were included, significant correlation from 0.47 -0.75 were also found among the trials.

Table 3. Correlation Table of RAVLT Trials 1 - 5, Immediate Post Interference and Delayed Trial.

\begin{tabular}{|c|c|c|c|c|c|c|c|}
\hline Source & Trial 1 & Trial 2 & Trial 3 & Trial 4 & Trial 5 & Immediate Post Interference & DelayedTrial \\
\hline Trial 1 & 1.00 & $0.67^{* *}$ & $0.66^{* *}$ & $0.57^{* *}$ & $0.58^{* *}$ & $0.49^{* *}$ & $0.58^{* *}$ \\
\hline Trial 2 & & 1.00 & $0.66^{* *}$ & $0.60^{* *}$ & $0.52^{* *}$ & $0.48^{* *}$ & $0.47^{* *}$ \\
\hline Trial 3 & & & 1.00 & $0.75^{* *}$ & $0.71^{* *}$ & $0.71^{* *}$ & $0.67^{* *}$ \\
\hline Trial 4 & & & & 1.00 & $0.71^{* *}$ & $0.70^{* *}$ & $0.70^{* *}$ \\
\hline Trial 5 & & & & & 1.00 & $0.72^{* *}$ & $0.72^{* *}$ \\
\hline Trial 6 & & & & & & 1.00 & $0.71^{* *}$ \\
\hline Delayed & & & & & & & 1.00 \\
\hline
\end{tabular}

$* *=$ Correlation is significant at 0.01 level.

On the other hand small to medium correlation were found on some trials on time taken to complete the task. Table 3 shows 
the trial time correlation.

Table 4. Correlation Table of RAVLT Trial Time for Trials $1-6$.

\begin{tabular}{lllllll}
\hline Source & Tt1 & Tt2 & Tt3 & Tt4 & Tt5 & Tt immediate Post Interference \\
\hline Trial Time 1 & 1 & -0.08 & $0.21^{*}$ & 0.05 & 0.04 & 0.09 \\
Trial Time 2 & & 1 & -0.01 & -0.04 & 0.05 & -0.04 \\
Trial Time 3 & & & 1 & 0.06 & $0.28^{* *}$ & 0.09 \\
Trial Time 4 & & & & 1 & $0.32^{* *}$ & $0.40^{* *}$ \\
Trial Time 5 & & & & & 1 & $0.29^{* *}$ \\
Trial Time 6 & & & & & & 1 \\
\hline
\end{tabular}

* = Correlation is significant at 0.005 level.

** = Correlation is significant at 0.01 level.

\subsection{Cardio Physiological Markers of RAVLT Performance}

The regression model was used to determine the possible contributions of cardiological markers (Systolic, diastolic and pulse) on RAVLT total performance. The standardized coefficient Beta were as follows: systolic $=0.13$, diastolic $=$ 0.09 and pulse $=0.06$. The overall $\mathrm{R}$ square was 0.02 while the model fit was not significant at $\mathrm{P} \leq 0.05 ; \mathrm{F}(3,113)=0.59$. For the delayed trial, the standardized co-efficient Beta were: systolic $=0.08$, diastolic $=0.09$ and pulse $=0.11$. The overall $\mathrm{R}$ square was 0.02 while the ANOVA model fit was not significant at $\mathrm{P} \leq 0.05 ; \mathrm{F}(3,113)=0.58$.

However for the recognition word list (RWL) trial the standardized co-efficient Beta were: systolic $=0.06$, diastolic $=0.09$ and pulse $=0.30$. The overall $\mathrm{R}$ square was 0.11 while the ANOVA model fit was significant at $\mathrm{P} \leq 0.05 ; \mathrm{F}(3,113)=$ 0.57 .

\subsection{Demographic Markers of RAVLT Performance}

Three demographic variables were examined in the study. Gender and illness status were main independent variables while age was put as a covariate. The findings of the study show no significant gender differences on the trials $1-5$ and immediate post interference trial except on trial 4, F $(1,122)$ $=7.91$ at $\mathrm{P} \leq 0.01$. However, significant gender differences were found on trial $\mathrm{B}, \mathrm{F}(1,122)=6.78$; Total Trial, $\mathrm{F}(1,122)$ $=4.41$ and delayed trial, $\mathrm{F}(1,22)=3.73$ all at $\mathrm{P} \leq 0.05$ level of testing. Their effect sizes were very small ranging from $0.01-0.06$.

Furthermore significant differences were found on illness status in all trials except trial B and trial 2. For the significant trials their $\mathrm{F}$ ratio were: Trial $1, \mathrm{~F}(2,122)=4.41$; Trial 3 , $\mathrm{F}(2,122)=7.21 ;$ Trial $4, \mathrm{~F}(2,122)=6.44 ;$ Trial $5, \mathrm{~F}(2,122)=$ 10.35; Trial 6, $\mathrm{F}(2,122)=7.59$, Total Trial, $\mathrm{F}(2,122)=7.11$ and Delayed Trial, $\mathrm{F}(2,122)=3.79$ all at $\mathrm{P} \leq 0.05$ level of significance. Their effect size calculated as partial eta ranged from $0.06-0.15$ showing a medium effect size. In addition, no significant interaction effects of gender and illness status were found among the sample population studied. However, age as a covariate showed significant difference on Delayed Trial, $\mathrm{F}(1,122)=3.87$ at $\mathrm{P} \leq 0.05$ level of significance, Trial $2, F(1,122)=4.57$ and $R W L, F(1,122)=3.92$ all at $P \leq 0.05$ level with effect size of $0.04-0.03$. Table 4 shows descriptive statistics of the demographic variables.

Table 5. Descriptive statistics of gender and illness status on RAVLT.

\begin{tabular}{|c|c|c|c|c|c|}
\hline Trials & Gender & Illness status & Mean & Std & $\mathbf{N}$ \\
\hline \multirow{10}{*}{ Trial 1} & \multirow{4}{*}{ Male } & $\mathrm{HC}$ & 6.23 & 1.63 & 43 \\
\hline & & PG & 4.53 & 1.55 & 15 \\
\hline & & Total & 5.79 & 1.76 & 58 \\
\hline & & $\mathrm{HC}$ & 6.84 & 1.74 & 57 \\
\hline & \multirow{3}{*}{ Female } & PG & 4.28 & 1.49 & 7 \\
\hline & & Total & 6.56 & 1.88 & 64 \\
\hline & & $\mathrm{HC}$ & 6.58 & 1.71 & 100 \\
\hline & \multirow{3}{*}{ Total } & PG & 4.45 & 1.50 & 22 \\
\hline & & Total & 6.19 & 1.86 & 122 \\
\hline & & $\mathrm{HC}$ & 8.02 & 1.81 & 43 \\
\hline \multirow[t]{9}{*}{ Trial 2} & \multirow[t]{3}{*}{ Male } & PG & 5.40 & 1.59 & 15 \\
\hline & & Total & 7.34 & 2.09 & 58 \\
\hline & & $\mathrm{HC}$ & 8.50 & 2.35 & 57 \\
\hline & \multirow[t]{3}{*}{ Female } & PG & 6.28 & 1.60 & 7 \\
\hline & & Total & 8.26 & 2.37 & 64 \\
\hline & & $\mathrm{HC}$ & 8.30 & 21.43 & 100 \\
\hline & \multirow[t]{3}{*}{ Total } & PG & 5.68 & 1.61 & 22 \\
\hline & & Total & 7.82 & 2.28 & 122 \\
\hline & & $\mathrm{HC}$ & 9.93 & 2.00 & 43 \\
\hline \multirow[t]{5}{*}{ Trial 3} & \multirow[t]{3}{*}{ Male } & PG & 5.66 & 1.63 & 15 \\
\hline & & Total & 8.82 & 2.67 & 58 \\
\hline & & $\mathrm{HC}$ & 10.21 & 2.16 & 57 \\
\hline & \multirow[t]{2}{*}{ Female } & PG & 6.14 & 2.60 & 7 \\
\hline & & Total & 9.76 & 2.53 & 64 \\
\hline
\end{tabular}




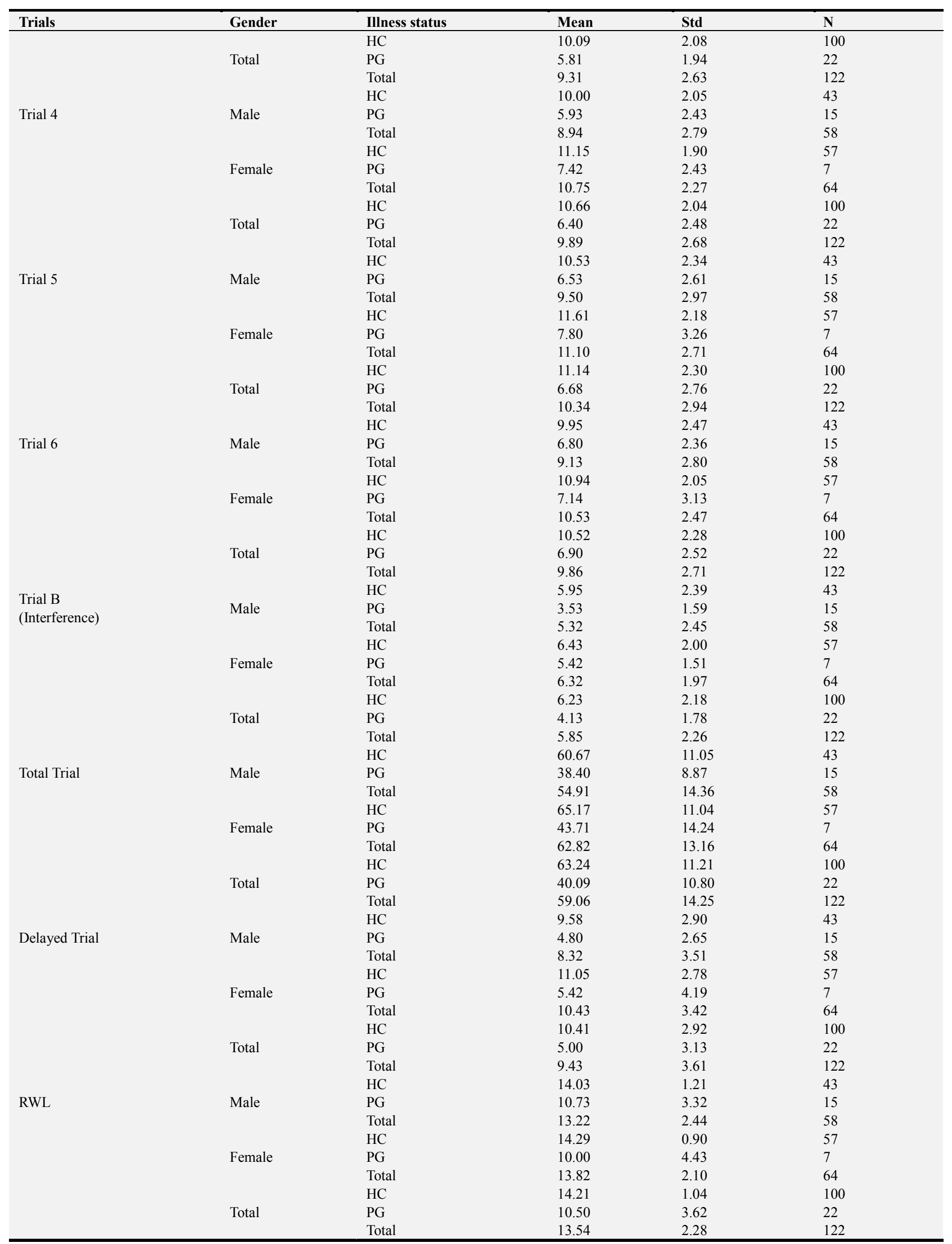




\section{Discussion}

The findings of the study show moderate to high inter-trial correlations of the Rey Auditory Verbal Learning Test among the participants studied. The analysis started with the Pearson product moment correlation of the first five trials. The range of the correlation scores showed high test-retest reliability of RAVLT among the samples studied. On further testing the post interference trial and delayed trial were included in the correlation matrix. The findings continued to show moderate to high correlation. The relationship between the first five trials and post interference trial showed that as the learning of the trials $1-5$ is improving, the correct response on post interference test is also improving. Conversely, the same was found for delayed trial when compared with trials $1-5$ and post interference trial respectively. Similar findings have been obtained in studies from other developing countries where the reliability of RAVLT has been tested. Magalhae, Malloy - Diniz and Hamdan [28] showed strong inter class coefficient and cronbach alpha coefficient as measure of reliability of the RAVLT in Brazil among young adult population. Our findings were also in line with other studies on the relationship level obtained from sum of trials $1-5$ (A1 - A5) RAVLT in different languages. For example Geffen and Geffen [29] got correlation of 0.77, Knight, McMahon, Skeaff and Green [30] got 0.79, while Magalha e et al [28] got 0.76 . Although the present study was on intertrial correlations of RAVLT administered in the usual clinical tradition, the other studies cited above gave some interval of days and calculated the mean scores and inter class correlation like the study of Magalhae et al [28]. In addition, our result was in line with the learning principle of primacy, recency and closeness effects. A closer look at table 1 showed that trials 1 and 2 had more similar correlation and trials 3, 4 and 5 had similar correlations.

The time taken to respond to the trials were also assessed. Table 3 showed small to medium correlations between some trials on RAVLT. The significant relationships were concentrated at the later trials (Trials 4, 5 and post interference). The first 3 trials showed no significant relationship on time taken to respond to the task. One could explain this phenomenon based on the assumption that the peak of the verbal learning was at trial 3. Additional trials now were relatively tasking on the brain which needed more effort to perform. Presumably at trial 3, the individual must have reached his/her highest level of verbal learning. Thus trials $4-5$ appear to be tasking on the individual as the person tries to add more words to the ones already learnt. In that case the time taken to perform appear to increase relatively within these trials.

Conversely, cardiological markers were used as predictors of RAVLT. The findings did not support the blood pressure measures and pulse as predictors of various measures of RAVLT. However the pulse rate index showed significant prediction of recognition word list. Ideally, several studies have associated cognitive decline with a poorly controlled blood pressure and pulse rate [31], [32], [33]. Persson, Lavebratt, Sundstrom and Fischer [34] showed that pulse pressure (PP) elevation moderated the effect of the functional $\mathrm{Val}^{158}$ met polymorphism on 15 years episodic memory (EM) trajectories leading to steep decline in Val carriers and accounting for various covariates with the potentials to affect individual differences in cognitive change. Zhou [35] showed the results of 6 years of follow up data from the Sacramento Area Latino Study of Aging (SALSA). The findings showed that higher pulse pressure was associated with longitudinal decline in cognitive function after adjusting for potential confounding variables and untreated individuals with high pulse pressure were at highest risk of cognitive impairments. The findings suggested an important role of optimal pulse pressure to preserve cognitive function in the elderly.

However, our present study differs from other literatures reviewed above as they were interested in the elderlies and middle aged samples. Our sample and interest were young adults. Very few studies have been done on the roles of cardio-functioning on episodic memory among this age bracket. The present study is an attempt to study the cardiomarkers of episodic memory among young adults. The findings thus implicated the pulse pressure and not systolic and diastolic measures as predictors of recognition word list of RAVLT. Pulse pressure and pulse wave velocity are known markers of arterial stiffness and have been associated with stroke, dementia and lowered levels of cognitive function. However our study did not find any predictive utility of systolic, diastolic and pulse pressure on recall phase of RAVLT, while small predictive utility was found of pulse pressure on delayed recall. The recognition procedure (RWL) of RAVLT measures how much was learned regardless of the efficiency of spontaneous retrieval.

According to Lezak et al [21] the recognition score examines the patient's capacity to discriminate when or with what other information a datum was learned. This technique may elicit evidence of the kind of disordered recall seen in patients with impaired frontal lobe functions who can learn readily enough but cannot keep track of what they have learned or organize it [21].

Our present findings showed significant gender differences on trial B (interference), total trial and post interference delayed trial. The females showed better recall on these trials. Past studies had always shown that females performed better than males on RAVLT [36], [2]. Similarly, our findings showed that age (as a covariate) had significant difference on delayed trial and recognition word list. Despite the difference of gender and age on RAVLT, the two demographic variables had little effect size on episodic memory. Contrary to other studies that had elderly population as their sample, our participants were young adults between the ages of $22-40$ years. The present findings imply that at young adulthood, age and gender may not contribute significantly to verbal memory performance among normal people and psychiatric population. Other contributory factors like pulse rate may be implicated. In addition, our findings showed significant differences between 
the normal population and psychiatric population on all the trials of RAVLT except trial B. This particular result showed the discriminate validity of the RAVLT in psychiatric diagnosis. Subsequently aside from the significant differences, their effect size ranged from small to medium effect size unlike gender and age variables. Previous findings have shown neuropsychological impairments among psychiatric population [37], [38] but to the best of our knowledge, no studies have focused on RAVLT as discriminant measure of psychotic depression in young adults. The importance and clinical relevance of this measure is that RAVLT is an easy to administer test of episodic memory and can be used as quick screening tool for psychiatric diagnostic purposes.

Our study had a major limitation with age of the participants as it relates to the normal and psychiatric samples. Their ages are not homogenous as the psychiatric sample significantly differed in age from the normal population. This may confound our result on the differences between the psychiatric and normal population. However, the effect size for age showed very little contribution of age on RAVLT performance. In addition our test of difference for age as a covariate showed that age only differed on delayed recall and recognition word list.

\section{Conclusion}

Overall the findings of the study showed that RAVLT is a reliable tool with discriminate validity among psychotic depressed population and normal samples. Further, our findings showed that pulse rate is a strong predictor of recognition word list in young adults.

\section{References}

[1] Magalhaes, S. S., \& Hamdan, A. C. (2010). The Rey Auditory Verbal Learning Test: normative data for the Brazilian population and analysis of the influence of demographic variables. Psychology \& Neuroscience, Vol. 3, No. 1, 85-91.

[2] Malloy - Diniz, L. F. M., Lasmar, V. A. P., Gazinelli, L. S. R., Fuentes, D., \& Salgado, J. V. (2007). The Rey Auditory Verbal Learning Test: applicability for the Brazilian elderly population. Revista Brasileira de Psquiatria, 29, 324-329.

[3] Spreen, O., \& Straus, E. (1998). A compendium of neuropsychological tests: administration, norms and commentary. New York: Oxford University Press.

[4] Vander Elst, W., Van Boxtel, M. P. J., Van Breukelen, G. J. P. \& Jolles, J. (2005). Rey's verbal learning test: normative data for 1855 Healthy participants aged 24-81 years and the influence of age, sex, education and mode of presentation. Journal of the International Neuropsychological Society, 11, 290-302.

[5] Mitrushina, M. N., Boone, K. B., Razani, J., \& D’ Elia, L. F. (2005). Handbook of normative data for neuropsychological assessment. New York: Oxford University Press.

[6] Loring, D. W., Strauss, E, Hermann, B. P., Barr, W. B. Perrine, K., Trenerry, M. R., Cheluna, G., Westerveld, M., Lee, G. P., Meador, K. J. \& Bowden, S. C. (2008). Differential neuropsychological test sensitivity to left temporal lobe epilepsy. Journal of the International Neuropsychological Society, 14, 394-400.

[7] Torres, I. J., Flashman, L. A., O’Leary, D. S., \& Andreason, N. C. (2001). Effects of retroactive and proactive interference on word list recall in schizophrenia. Journal of the International Neuropsychological Society, 7, 481-490.

[8] Bowler, D. M., Limoges, E., \& Mottron, L. (2009). Different verbal learning strategies in autism spectrum disorder: Evidence from the Rey Auditory Verbal Learning Test. Journal of Autism and Developmental Disorders, 39, 910 915.

[9] Hamdan, A. C., \& Bueno, D. F. A. (2005). Relacoes entre controle executive e memoria episcodica verbal no compromentimento cognitivo leve e na demencia tipo. Alzheimer Estudos de Pscicologia, 10, 63-71.

[10] Duff, K., Schoenberg, M. R., Scott, J. G., \& Adams, R. L. (2005). The relationship between executive functioning and verbal and visual learning and memory. Archives of Clinical Neuropsychology, 20, 111-122.

[11] Furentos, D., Malloy-Diniz, L. F., Camargo, C. H. P. \& Cosena, R. M. (2008). Neuropscologia: teoria e practica. Porto Alegre: Artmed.

[12] De la Torre, J. C. (2002). Alzheimer's disease as vascular disorder: nosological evidence. Stroke, 33, 1152-1162.

[13] Kaffashian, S., Dugravot, A, Elbaz, A., Shipley, M. J., Sabia, S., Kivimaki, M., \& Singh, S., Kivimaki, M., \& SinghManoux, A. (2013). Predicting cognitive decline: A dementia risk score vs the Framingham vascular risk scores. Neurology, Vol. 80, No. 14, 1300-1306.

[14] Geaney, D. P., Soper, N., Shepstone, B. J. \& Cowen, P. J. (1990). Effect of central cholinergic stimulation on regional cerebral blood flow in Alzheimer's disease. Lancet, 335, 1484-1487.

[15] Van Beek, A. H \& Classen, J. A. (2011). The cerebrovascular role of the cholinergic neural system in Alzheimer's Disease. Behaviour Brain Research, 221, 537-542.

[16] Picano, E., Bruno, R. M., Ferrani, G. F. \& Bouncelli, U. (2014). Cognitive impairment and cardiovascular disease: so near, so far. International Journal of Cardiology. http://dx.dio.org/10.1016/j.ijcard.2014.85.004.

[17] Pizza, V., Agresta, A., D’Acunto, C. W., Festa, M., Capasso, A. (2011). Neuroflammation and ageing: Current theories and an overview of the data. Review on Recent Clinical Trials, 6 , 189-203.

[18] Waldstein, S. R. (1995). Hypertension and neuropsychological function: A life perspective. Experimental Aging Research, $21,321-352$.

[19] Waldstein, S. R. \& Katzel, L. I. (2001). Hypertension and cognitive function. In S. R. Waldstein \& M. F. Elias (Eds.), Neurophychology of cardiovascular disease. Mahah, N. J: Erlbaum.

[20] Waldstein, S. R., Manuek, S. B., Ryan, C. M. \& Muldon, M. F. (1991). Neuropsychology correlates of hypertension: Review and methodologic considerations. Psychological Bulletin, 110, $451-468$. 
[21] Lezak, M. D., Howieson, D. B., Bigler, E. D. \& Tranel, D. (2012). Neuropsychological Assessment (5ed.). Oxford: Oxford University Press.

[22] Brown, J. H., Kinsella, G. J., Ong, B., \& Volvels, L. (2000). A Study of Performance of delayed intentions in multiple sclerosis. Journal of Clinical and Experimental Neuropsychology, 22, 418-429.

[23] Butters, N., Wolfe, J., Granholm E., \& Martone, M. (1986). An assessment of verbal recall, recognition, and fluency abilities in patients with Huntington's disease. Cortex, 22, 1132 .

[24] Bigler, E. D., Rosa, L., Schultz, F. et al (1989). Rey Auditory Verbal Learning and Rey-Osterrieth Complex Figure Design Performance in Alzheimer's disease and closed head injury. Journal of Clinical Psychology, 45, 277-280.

[25] Ferman, T. J., Smith, G. E., Boone, B. F. et al (2006). Neuropsychological differentiation of dementia with lewy bodies from normal ageing and Alzheimer's disease. The Clinical Neuropsychologist, 20, 623-636.

[26] Zhou, A. \& Jia, J. (2009). Different cognition between mild cognitive impairment due to small vessel disease and mild cognitive impairment of Alzheimer's disease origin. Journal of the International Neuropsychological Society, 15, 898-905.

[27] Tierney, M. C., Moineddin, R. \& McDowell, I. (2010). Prediction of all cause dementia using neuropsychological tests within 10 and 5 years of diagnosis in a community based sample. Journal of Alzheimer's Diseases, 22, 1231-1240.

[28] Magalhaes, S. de sous, Malloy-Diniz, L. F., \& Handas A. C. (2012). Validity convergent and reliability test-retest of the Rey-Auditory Verbal Learning Test. Clinical Neuropsychiatry, 9, 129-137.

[29] Geffen, G., \& Geffen, L. (2000). Auditory Verbal Learning Test (AVLT): computerized scoring program and population norms. Acer Press: Australia.

[30] Knight, R. G., McMahon, J., Skeaff, C. M., \& Green, T. J.
(2007). Reliable change index scores for persons over the age of 65 tested on alternate forms of the Rey AVLT. Archives of Clinical Neuropsychology, 22, 513-518.

[31] Waldstein, S. R. (2003). The relation of hypertension to cognitive function. Current Direction in Psychological Science, 12, 9-13.

[32] Waldstein, S. R., Rice, S. C., Thayer, J. F., Najjar, S. S., Scuteri, A., \& Zonderman, A. B. (2008). Pulse pressure and pulse wave velocity are related to cognitive decline in the Baltimore Longitudinal Study of Aging. Hypertension, 51, 99-104.

[33] Person, N., Vitanen, M., Almkvist, O. \& Wahlin, A. (2013). A principal component model of medical health: Implications for cognitive deficits and decline among adults in a population based sample. Journal of Health Psychology, 18, 1268-1287.

[34] Person, N., Lavebratt, C., Sundstrom, A., \& Fischer, H. (2016). Pulse pressure magnifies the effect of COMT Val ${ }^{158}$ met on 15 years episodic memory trajectories. Frontiers in Aging Neuroscience, 8, 34.

[35] Zhou, X. (2010). Blood pressure, pulse pressure and antihypertensive treatment: Association with cognitive decline and dementia in elderly Mexican - Americans. A Doctoral Dissertation University of Michigan, Epidemiological Science.

[36] Bolla - Wilson, K., \& Bleecker, M. L. (1986). Influence of verbal intelligence, sex, age and education in the Rey Auditory Verbal Learning Test. Developmental Neuropsychology, Vol. 2, No. 3, 203-211.

[37] Seidman, L. J., Gilliliano, A. J. \& Meyer, E. C. Addington, J., Cadenhead, K. S., Cannon, T. O. et al (2010). Neuropsychology of the prodrome to psychosis in the NAPLS consortium: Relationship to family history and conversion to psychosis. Archives of General Psychiatry, 67, 6, 578-588.

[38] Fleming, S. K., Blasey, C. \& Schatzberg, A. F. (2004). Neuropsychological correlates of psychotic features in major depressive disorders: a review and meta analysis. Journal of Psychiatric Research, Vol. 38, 1, 27-35. 